\title{
Antonio de Cabezón y el Comienzo de la música didÁctica para tecla con VALOR ARTÍSTICO
}

\author{
Antonio de Cabezón and the Beginning of the Didactical Keyboard Music with \\ AN ARTISTIC VALUE
}

Stephan Schmitt

Hochschule für Musik und Theater, München

\begin{abstract}
Resumen:
Con las Obras de música para tecla, arpa y vihuela de Antonio y Hernando de Cabezón tenemos en las manos uno de los ejemplos más antiguos de método para instrumentos, que reúne la lógica didáctica con el alto nivel artístico. Fuera de eso, en las composiciones "para principiantes", no se cumple mecánicamente el principio "de lo sencillo a lo dificil”, sino que Cabezón, en primer plano, concibe las posibilidades, esboza los espacios de juego y las libertades, en vez de traspasar demasiado sistemáticamente un espacio limitado por reglas. La distancia de los métodos contemporáneos para tecla, respecto a la calidad, justifica con ello, la valoración, que con este método se nos ha puesto en las manos, el primer ejemplo de música didáctica para tecla con valor artístico - 150 años antes de las correspondientes obras de Johann Sebastian Bach y 250 años antes del Álbum para la juventud de Robert Schumann.
\end{abstract}

Palabras clave:

Antonio de Cabezón; didáctica; pedagogía; método instrumental; tecla; órgano; nivel artístico; ceguera; improvisación.

\begin{abstract}
:
Obras de música para tecla, arpa y vihuela by Antonio and Hernando de Cabezón represents one of the earliest examples of an instrumental method that combines didactic logic and a high level of artistry.

In addition, these compositions "for beginners", do not follow the principle "from the simple to the difficult" in a mechanical way. Instead, Cabezón provides alternatives, outlines experimental possibilities, and leaves room for liberties, instead of simply striding systematically through material that is limited by rules.

The discrepancy in quality between his method and keyboard methods of his contemporaries, justifies the appraisal that this method is first example of didactic music for keyboard instruments with artistic value - 150 years before the corresponding works of Johann Sebastian Bach and 250 years before Robert Schumann's Album für die Jugend.
\end{abstract}

Key Words:

Antonio de Cabezón; didactics; pedagogy; instrumental method; keyboard; organ; artistic level; blindness; improvisation. 
El objeto del presente trabajo son las composiciones de Antonio de Cabezón, que muestran intenciones didácticas en la técnica compositiva y en la disposición. Hernando define estas piezas, recopiladas en las Obras, explícitamente, como composiciones para principiantes. A excepción de los versos sobre el saeculorum y de los fabordones, son a dos o tres voces. La intención didáctica de ambas composiciones del Libro de Cifra Nueva de Luys Venegas de Henestrosa es deducible también de su situación al comienzo de la publicación, dentro de las composiciones a dos o tres voces. Los citados fabordones, que, en las Obras..., siguen a las composiciones introductorias y técnicamente más fáciles, no son objeto de este estudio, aunque también, en este caso, se manifiesta una progresión de lo sencillo a lo más difícil, cosa que, sin embargo, es una práctica usual también en las variaciones sin intención didáctica alguna. El aumento de virtuosismo en la técnica de ejecutar y complejidad técnico-compositiva, en este caso, depende de la concepción dramática y, a lo sumo, ofrece un paralelismo en intenciones didácticas.

La tarea que me he propuesto es investigar algunas de las mencionadas composiciones respecto a su material temático y elaboración, a fin de poder sacar conclusiones con vistas a la cuestión de su función didáctica y a las complejas deliberaciones sobre el rango artístico de estas composiciones dentro del corpus total. La relación de declaraciones analítico-estructurales con juicios valorativos -necesariamente subjetivos-, que se basan no sólo en las propias declaraciones, sino también en las de otros científicos, dará por resultado que dichas composiciones representan los primeros ejemplos de música didáctica de tecla con valor artístico, y esto, casi 150 años antes de las Invenciones y Sinfonías y demás composiciones pertinentes de J. S. Bach, que, hasta ahora, eran consideradas como el principio de la literatura didáctica de nivel artístico.

\section{LA PRÁCTICA MUSICAL DE LA ÉPOCA, ENTRE IMPROVISACIÓN Y COMPOSICIÓN}

La notación musical para tecla, en sus inicios, se distingue de la música vocal de la época y de la música de la época de Bach, entre otras cosas, porque es música improvisada, cuya transcripción posterior ${ }^{1}$ persigue intenciones didácticas, es decir, se trata de una instrucción para la improvisación, que aconseja ejemplos bien logrados, para tomarlos por modelo. "El Fundamentum, sin embargo, enseña la ejecución, pero no la composición, si bien los criterios compositivos no pueden quedar fuera de consideración. La meta de los discípulos de Hofhaimer no era componer como él, sino tocar como él"2. Lo que Kugler constata a propósito de la práctica alemana también vale, con restricciones, fuera de Alemania y hasta el siglo XVI. Esto coincide con el hecho de que las notaciones en España, aunque no sólo aquí, eran realizadas por medio de una "escritura de tañer" (Griffschrift), ya que en esto se manifesta el aspecto táctil, técnicoejecutivo, aunque la construcción sonora y temática apenas es reconocible. 105.

1 Schrade, Leo: Die handschriftliche Überlieferung der ältesten Instrumentalmusik. Lahr, Moritz Schauenburg, 1931, p.

2 Kugler, Michael: Die Musik für Tasteninstrumente im 15. und 16. Jahrhundert. Wilhelmshaven, Heinrichshofen, "Taschenbücher zur Musikwissenschaft, 4”, 1975, p. 91. 
La dependencia de la música de tecla, en España, del "arte de composición de la polifonía vocal”3 y, a la vez, de la técnica imitativa, se pierde, sin embargo, durante el desarrollo musical hacia composiciones perfiladas y específicas para tecla, que se entendían como "obras", es decir, como composiciones que se ofrecían tan acabadas y perfectas que no eran apreciadas sólo como modélicas y dignas de ser imitadas, sino que representaban una música incomparable, que valía la pena conservar y legar a la posteridad. En la España del siglo XVI, este desarrollo puede reconocerse ejemplarmente en la colección de las Obras... de Cabezón.

\section{OBRAS DIDÁCTICAS}

Los Fundamenta del siglo xv, como los otros tratados del siglo XVI, ofrecen casi un Studium genera$l e$, que cubre campos teóricos y prácticos: enseñan teoría de la música en un sentido amplio, incluyendo organología, contrapunto, improvisación, composición y ornamentación. En el marco de estos tratados se encuentran composiciones ejemplares, que permiten estudiar la transición de la improvisación a la composición. Ciertamente, la Declaración de instrumentos musicales de Bermudo y el Arte de tañer fantasía de Tomás de Sancta María constituyen los tratados más conocidos de su tiempo. Pero, de hecho, el aspecto técnico-ejecutivo-didáctico apenas desempeña un papel en ambos casos: "Bermudo [...] trata esencialmente del género vocal [...] San[c]ta María, por el contrario, se interesa por la práctica contem-

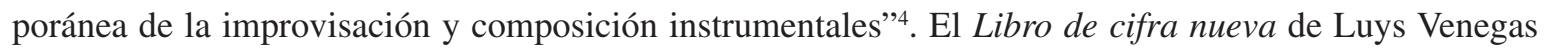
de Henestrosa, así como las Obras de Cabezón, reducen la instrucción teórica al mínimo; no obstante, ofrecen composiciones ejemplares, que en el caso de las Obras, ascienden incluso a más de una tercera parte de la colección entera (30 de un total de 85 composiciones).

\section{LA CEGUERA DE CABEZÓN Y SUS CONSECUENCIAS PARA LA CONCEPCIÓN Y NOTA- CIÓN MUSICAL DE LAS OBRAS...}

Surge una situación particular, respecto a la cuestión de improvisar o componer, cuando el autor es ciego, como era el caso de varios músicos antiguos: Landini, Paumann, Schlick y, también, Antonio de Cabezón. Su capacidad de memorizar debe ser mucho más marcada que la de músicos que pueden leer partituras. Para fijar por escrito sus improvisaciones o composiciones, los ciegos precisan un ayudante, ante el que ejecutan la música a cámara lenta y con muchas interrupciones. La idea de un improvisador,

3 Ibíd., p. 142.

4 Roig-Francolí, Miguel Á.: "En torno a la figura y la obra de Tomás de Sancta María: Aclaraciones, evaluaciones y relación con la música de Cabezón”, en Revista de Musicología, 15 (1992) pp. 55-85. Vid. también HäFNER, Roland: Die Entwicklung der Spieltechnik und der Schul- und Lehrwerke für Klavierinstrumente. Munich, Musikwissenschaftliches Seminar, "Schriftenreihe des musikwissenschaftlichen Seminars der Universität München, 2", 1937, p. 26.

5 Anglès, Higinio: La música en la Corte de Carlos v, con la transcripcion del Libro de Cifra Nueva para tecla, harpa y vihuela de Luys Venegas de Henestrosa (Alcalá de Henares, 1557). Barcelona, csic, "Monumentos de la Música Española, 2", 1944, pp. 158 y ss. 
que toca de improviso y espontáneamente inspirado, es de todos modos una ficción, ya que sin la ayuda de pasajes preformados, de fórmulas "armónicas" y melódicas o material ya existente, que pueda modificarse dentro de un determinado marco, la improvisación, al menos en aquellos tiempos, es inimaginable. Esto, desde luego, significa también que pasajes improvisados que el músico evaluaba como perfectos se reutilizaban en futuras improvisaciones, tras un proceso de organización y reorganización del material musical en la mente del músico ciego. Cuando, no obstante, como en el caso de Cabezón, la música es casi sin excepción polifónico-imitativa, se puede tener por seguro que el proceso de la composición ya estaba terminado en el momento en que la pieza musical se ponía por escrito. Las piezas puestas por escrito de un organista ciego, por consiguiente, deberían ser consideradas en su totalidad como composiciones y, con esto, como "obras". Con tal motivo, me atrevo a formular la tesis siguiente: En el caso de transcripciones de improvisaciones de un ciego, se trata siempre de pre-escritura, salvo que se transcriba una grabación, cosa que no ha sido posible hasta el siglo xx. La diferencia entre la post-escritura de una improvisación y la pre-escritura de una composición debió ser mínima, también entonces, en el caso de músicos que podían ver, ya que la imprenta era dispendiosa y cara.

Puesto que, con ciegos, la concepción y redacción de una composición tienen que realizarse en la mente -es de poca importancia si esto se efectúa con el instrumento o con ayuda del oído interno- y, porque, como ya he dicho, la capacidad de memorizar de un músico ciego es mucho mayor que la de uno que puede ver, se puede concluir, que también las piezas cortas y sencillas, redactadas para la enseñanza y que además se apuntaban, han sido minuciosamente elaboradas y muy pulidas. Con otras palabras: un músico ciego, que tiene preparado un repertorio amplio de piezas acabadas en la memoria, que muy probablemente trabaja constantemente más piezas en la mente, es decir, que se encuentra en un proceso permanente de experimentación, reorganización y perfeccionamiento, podrá presentar obras optimizadas en todos los aspectos y acabadas hasta el último detalle, en el momento de fijar su música en el papel. El testimonio, en el caso de Cabezón, que se trata sólo de "migajas que caýan de su mesa", es, por cierto, una humilde constatación (understatement) de su hijo.

\section{INTENCIONES DIDÁCTICAS DE ANTONIO DE CABEZÓN}

No siempre se distingue con precisión entre los conceptos pedagógico y didáctico; la causa entre otras cosas, es, que los conceptos están sujetos a un proceso histórico de modificación y ni siquiera, dentro de un espacio lingüístico, pueden encontrarse definiciones generalmente aceptadas. Por consiguiente, es necesario precisar el significado en cada momento de su empleo. En el marco del presente artículo, será la pedagogía el concepto general de cada cambio humano, orientado a la educación y formación, como también su investigación científica. La didáctica será el aspecto del cambio del ser humano, que, empujado por la enseñanza y conseguido por medio del aprendizaje, se dirige, por lo tanto, a la ampliación de conocimientos, faculdades y habilidades. En la preparación de obras para enseñar, se trata pues de literatura didáctica. 
Cabezón tenía experiencia como profesor. Dió lección al príncipe Felipe y a las infantas Doña María y Doña Juana y, desde luego, también a sus propios hijos. En el Proemio (p. 22) ${ }^{6}$, Hernando indica, que Cabezón aceptó también discípulos pobres, es decir, los que no podían pagar nada: «No se alçó con este talento maravilloso, ni dexó de comunicarlo a quantos él pudo enseñar que fuessen capazes dello, aunque fuesen muy pobres. "Y en otro lugar (Proemio, p. 23): “[...] no son más que las lectiones que él dava a sus discípulos, las quales no eran conforme a lo que sabía el Maestro, sino a la medida de lo que ellos podían alcançar y entender." Terni ${ }^{7}$ señala el "senso umano" de sus lecciones y sigue: "[...] l'amore per gli allievi, specialmente per i più poveri, il non scoraggiare i meno dotati, incitandoli ad arrivare fin dove lo comportano le loro possibilità e l'invito a mettersi nelle mani di un buon insegnante." Incluso extrae del proemio un credo didáctico - hoy en día se diría que "va a buscar al [alumno] en el punto donde hay que suponer que está, según la espera auditiva y su capacidad receptiva" "Si direbbe che unico problema del Cabezón era la maniera di trasmettere il suo modo di suonare ai discepoli, a seconda delle proprie capacità."

Ya Häfner" ve "en el puro predominio externo de los ejemplos prácticos frente a las meras indicaciones escritas de tocar la actitud avanzada frente a Bermudo y San[c]ta María. Por consiguiente, no es muy arriesgado considerar las «Obras...» de Cabezón como método de tecla - naturalmente, no en el sentido moderno. Por una parte, debido a la distribución de la materia en progresiva dificultad, por otra, a causa de la misma materia, que ahora muestra una inclinación más clara a la ejecución autónoma en el teclado." Las «Obras...» seguramente no son concebidas en su totalidad como método docente; sin embargo, Hernando ha antepuesto a la colección de «Obras...» un método de tecla, por medio de breves informaciones teóricas, al principio, y por la inclusión de Dúos para los principiantes, de obras de a tres para principiantes, de obras de a quatro y de favordones de todos los ocho tonos ${ }^{10}$, en total de las primeras 30 composiciones. ,...conozco [...] en aver querido juntar en este libro algunas cosas que él dio de lición a sus discípulos, por no haber sido cosa que él uviese hecho de próposito para este fin...“ (Proemio, p. 29)

Me parece dudoso -como piensa Kugler ${ }^{11}$ - que la sistemática en el listado de posibilidades de elaborar los versos se debe a consideraciones didácticas. También los demás Kyries, en el segundo tomo (51-57), se presentan sistemática e íntegramente concebidos y realizados en los ocho tonos.

Queda por constatar, que las intenciones didácticas que se formulan en el proemio y en el título de los tres primeros grupos, no resultan o, no exclusivamente, de un estratégico cálculo mercantil, sino

6 Referencias a un proemio o a ejemplos musicales siempre se refieren a la edición en tres volúmenes "Obras de música para tecla, arpa y vihuela" de Felipe Pedrell, según la nueva edición de Higinio Anglés, Barcelona 1966.

7 Terni, Clemente: "Ricordo de Antonio de Cabezón", en: Chigiana 23, 1966, pp. 25-41.

8 Ehrenforth, Karl Heinz: Verstehen und Auslegen. (Schriftenreihe zur Musikpädagogik), Frankfurt am Main, 1971. p. 5.

9 HäFNER, Roland: Die Entwicklung der Spieltechnik und der Schul- und Lehrwerke für Klavierinstrumente, Munich, Musikwissenschaftliches Seminar, 1937. (Schriftenreihe des musikwissenschaft-lichen Seminars der Universität München; 2), p. 26 y ss.

10 “...y los que más supieren, los servirán con los favordones que adelante vienen glosados para psalmear”, p. 31 de la parte musical)

11 "Gracias a su sencillez espartana y su transparencia, los versos para principiantes hacen evidente el principio del canto llano en redondas, con el que el alumno debe ejercitarse en primer lugar. Conforme a eso, la agrupación de a cuatro tiene su origen en el propósito didáctico de poner de relieve el principio estructural - canto en el tiple, contralto, tenor y bajo.” KuGLER, Michael: Die Musik für Tasteninstrumente... op. cit., p. 148 y ss. 
que pueden ser seleccionadas por la disposición y por la técnica compositiva. Por decirlo así, el primer Dúo empieza ab ovo a dos voces, punctus contra punctum, en valores largos, en una especie de cursillo rápido, pues ya, en el compás 19 , Cabezón pasa a las notas negras y, en el compás 30 a la imitación. Los tres primeros Dúos no llevan canto llano litúrgico y ofrecen de esta manera modelos de ejercicio para el tiento. El Dúo 4 y los siguientes (a excepción del Dúo 9) se basan en himnos, que van acompañados por imitaciones, con lo que se introduce en el canto llano elaborado. Igualmente, en el caso de las piezas para principiantes a tres o cuatro voces, una voz se encarga del canto llano y, en el caso de los ejecicios de ornamentación de los fabordones, se presupone la sucesión de acordes. Hasta aquí, queda siempre un apoyo, que acompaña al alumno en sus propios intentos de improvisar o componer. A la vez, no se le exige demasiado en cuanto a su técnica de ejecución, sino que se ve guiado a través de piezas cortas y sencillas hasta otras más extensas, desde las de valores largos a otras de valores más cortos, de pasajes homófonos a polifónicos. En el transcurso de las piezas a tres voces puede verse claramente, que se fomenta sutilmente la independencia de las manos: al principio son raros, y sólo breves, los movimientos de negras o corcheas en ambas manos. En los dos himnos 12 y 14, esto puede verse casi permanentemente.

\section{ANÁLISIS}

\section{Los Dúos}

\section{Dúo I de los Dúos para principiantes}

Ya el primer ejemplo de la colección es una obra maestra de la economía compositiva y, en eso, un botón de muestra en la relación dialéctica entre unidad y variedad de la polifonía imitativa. Cabezón desarrolla de un mínimo de material básico inicial -en lo esencial se trata de ejercicios de escalas- un máximo de efecto, es decir, de variedad de formas, que se relacionan mutuamente. Los sujetos están construidos de tal manera, que es posible hacer estrechos con ellos, que pueden servirse a si mismos de contrapunto y, con esto, dar la impresión de como si se tratase de una parte de un canon. Hay que tener en cuenta, que con sujetos cortos de pocos y pequeños saltos, la cantidad de posibles formas es menor en comparación a sujetos amplios y espaciosos. La cercanía y la coincidencia con "formas primarias", por ejemplo, con trozos de escalas o simples repeticiones, es, de este modo, mucho más probable. Con esto existen, no obstante, más posibildades de «estrechar» las formas, de combinarlas con escasa modificación y así reunirlas en una textura más densa.

La intención didáctica se muestra en el Dúo inicial, no solo en el número limitado de dos voces y en la trasparente estructura -las nuevas entradas están casi siempre separadas de lo anterior por pausas-, sino también en una introducción, que, empezando con redondas (punctus contra punctum) progresa hacia las blancas y las negras. Con esta aceleración interna, ofrece al alumno, oyente como ejecutante, la ocasión de orientarse, y esto, según el principio didáctico "de lo simple a lo dificil", refrendando con esto la progresión en el tempo, como también el progreso de lo sencillo a lo complejo. 
La escala, que, durante largo tiempo, ha sido el comienzo en toda la enseñanza de música instrumental y de teoría musical general - por así decirlo, la exposición del material disponible en su forma más sencilla - está tematizada en el primer Dúo, por consiguiente, como objeto de la lección, en doble sentido y, por lo tanto, como algo omnipresente. También sirve de base a la secuencia en los compases 14-17 e insinúa, en los compases 19 y 30, un propio sujeto, que aparece en el tiple (c. 19) y en el bajo (c. 25).

\section{Ejemplo 1.}

\section{Dúo I}

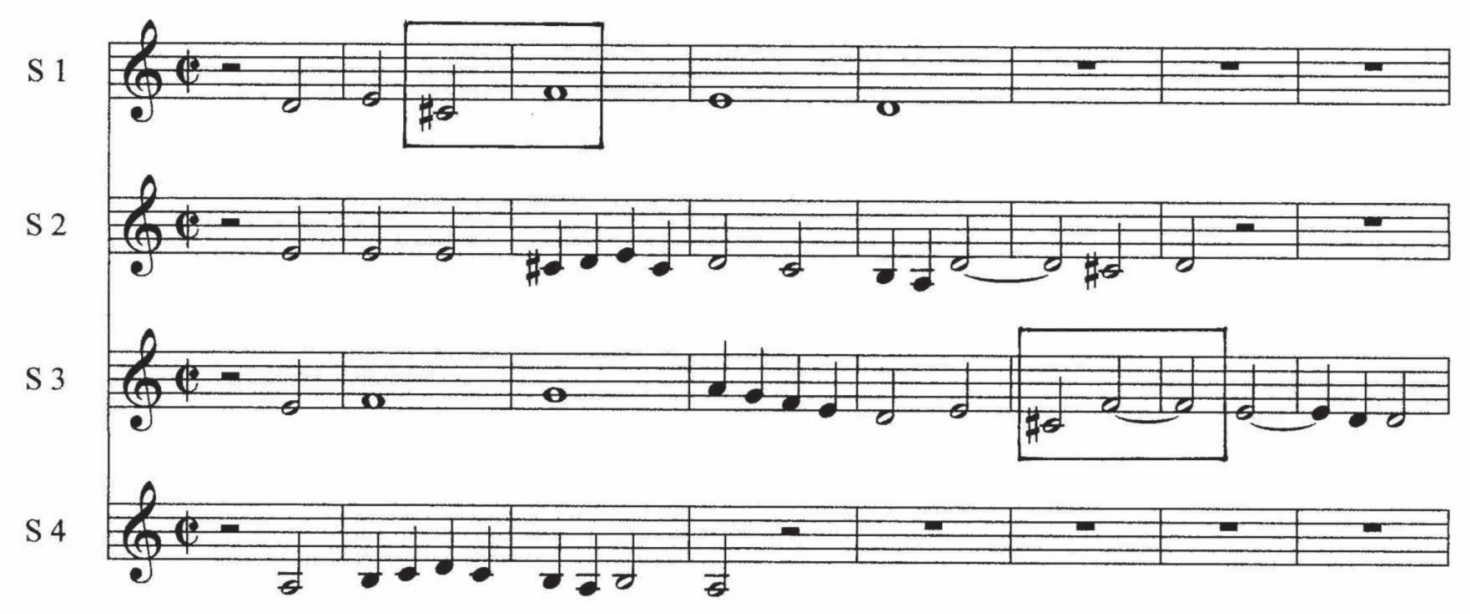

Los sujetos 1, 3 y 4 son afines por su forma arqueada, que se realiza, a la vez, por movimientos de escala. En esto, el tercer sujeto cita al primero al pie de la letra, sólo un poco desplazado temporalmente. El sujeto 2 se sale un poco de lo corriente, ya que comienza con repeticiones (que, junto a los pasos y saltos, es la tercera posibilidad fundamental de moverse la melodía, y algo fácil de comprender para el principiante). El parentesco del segundo sujeto con el primero es fácilmente reconocible gracias al idéntico comienzo rítmico y a las notas esenciales. También Howell ${ }^{12}$ describe este principio de composición en su detallado y ejemplar análisis del Tiento XVII del Libro de cifra nueva (aquí nr. XLIV): “[...] all the subjects are derived from a common source and all are linked with the opening subject." Incluso, cuando la pieza no es "en sentido estricto, monotemática", esta técnica, a pesar de esto, señala hacia dicha dirección y es un principio de composición, genuinamente instrumental, ya que, en la polifonía vocal, la unión al texto dificulta la semejanza de las formas.

12 Howell, A. C.: „Cabezón: An Essay in Structural Analysis“, in: MQ 50,1964, p. 20 
Cabezón usa, con pocas excepciones, solamente saltos pequeños como de tercera y cuarta en esta pieza para principiantes. La destacada cuarta disminuída ofrece, no obstante, algún brillo, después de ser preparada en la introducción (c. 27s.) cuando aparece explícitamente en los sujetos 1 y 3. A Cabezón le gustan tales momentos de sorpresa y, sobre todo, este intervalo (ve también: Tiento III del primer tono, Tiento IV del segundo tono) y, significativamente, no quiere prescindir de esto en la pieza más sencilla de la colección. También Kastner alude a esto: “Cabezón’s forms do not have a Baroque or Classical regularity, but rather a capricious individuality."

Howe $1{ }^{13}$ llama la atención sobre una peculiaridad estructural, que puede observarse en muchas composiciones de Cabezón y que dificulta la articulación: “[...] attempt to divide Cabezón's tientos into sections is often a baffling task, especially to the organist [...] Sectional divisions must be located at the places where the thematic material changes, and not necessarily at these spots where the strongest cadential formation occurs." Esta borrosidad de la estructura no se encuentra en las piezas para principiantes, probablemente, debido a la intención didáctica. Las claúsulas están al final de las secciones del sujeto, son claramente perceptibles y terminan en unísonos y en intervalos de octava sobre la nota fundamental (c. 5/6, 17/18, 24/25, 28/29, 46/47, 77/78, 81/82) o sobre la quinta (c. $39 / 40,55 / 56)$.

\section{Dúo II de los Dúos para principiantes}

Citaré otras composiciones sólo en vista de sus peculiaridades. Sin embargo, todas muestran aspectos inesperados y extraordinarios, y son singulares a su manera. Los compases 1-17 del Dúo II (sujeto $1 \mathrm{y}$ una parte de sujeto 2) presentan un canon riguroso a distancia de tres compases. El canon como punto de encuentro de toda la música imitativa, como - por decirlo así - meta inalcanzada e inalcanzable de toda la composición de fuga, ocupa aquí un espacio sorprendentemente grande. El pasaje también da que pensar, respecto a la habilidad de Cabezón como músico ciego. Pues la composición de un canon, para que no sea considerablemente simple, apenas es concebible sin la posibilidad de usar la escritura musical.

13 Howell, A. C.: Cabezón: An Essay in Structural Analysis“, in: MQ 50,1964, p. 21. 


\section{Ejemplo 2.}

\section{Dúo II}

S 1

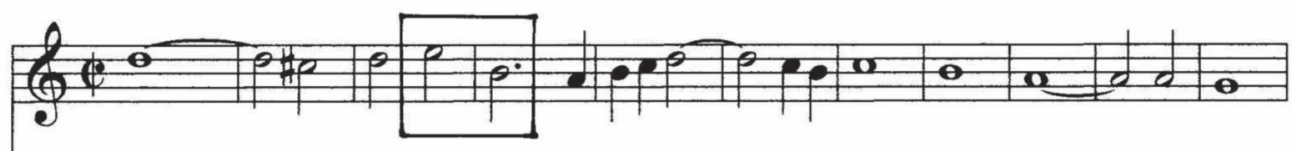

S 2

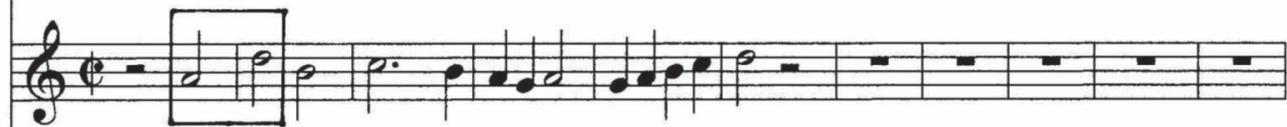

S 3

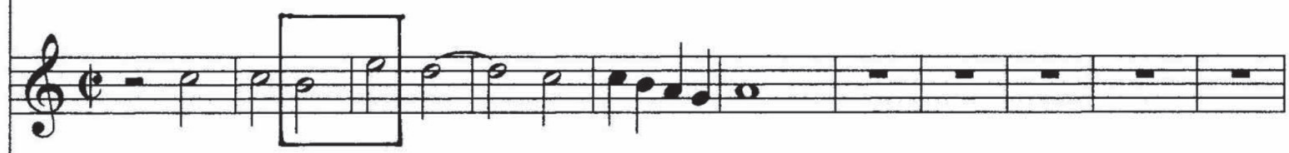

S 4

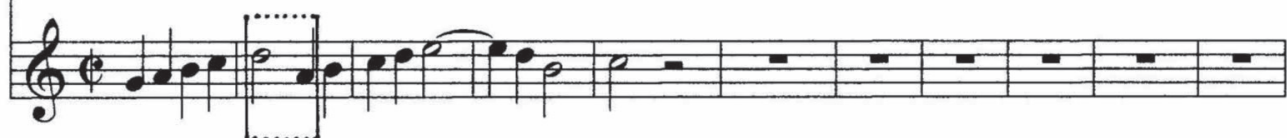

El parentesco de los cuatro sujetos ya no es tan marcado como en la primera composición. Parece, sin embargo, como si Cabezón quisiera poner en el centro el salto de cuarta justa y contrastarlo con el movimento gradual que, por lo demás, sigue prevaleciendo. También los fragmentos de escalas juegan aquí un papel importante; el sujeto 4 consta casi exclusivamente de tales figuras, que ya forman parte del sujeto 2.

\section{Los Trios}

\section{Kyries de Nuestra Señora: Rex virginum I-III}

En los tres Kyries de Nuestra Señora (nr. X del primer volumen), Cabezón añade al canto llano de la voz intermedia una voz superior y otra inferior, que - como succede generalmente en este tipo de composición sobre un canto llano - no muestran relación motívica alguna con la melodía gregoriana. El Kyrie 1 es homófono, los Kyries 2 y 3 comienzan con imitación. 


\section{Ejemplo 3.}
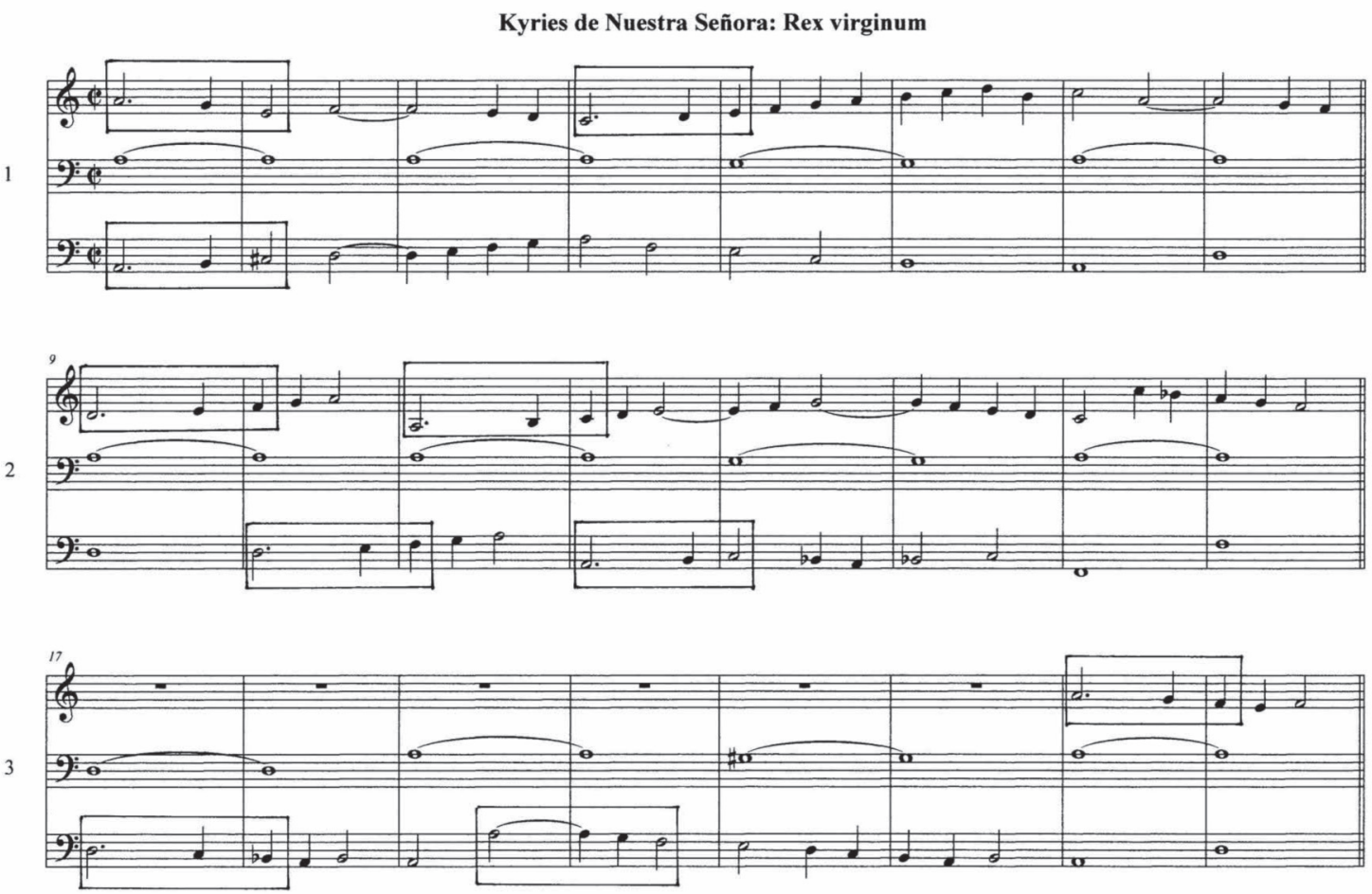

La comparación de los primeros compases muestra, que Cabezón ha concebido las composiciones en forma de ciclo: La blanca con puntillo, seguida de una negra, aparece por todas partes, en el Kyrie 2, llevada incluso en forma de canon. Este motivo, en dirección ascendente o descendente, es la célula esencial del siguiente trabajo motívico, pues las tres piezas constan de esta célula y de su continuación en forma de fragmentos de escalas. Las piezas están entretejidas compactamente de esas formas melódicas primarias de tal modo, que apenas se encuentra un movimiento no-motívico. Cabezón introduce el movimento simultáneo de negras en las voces exteriores, sólo excepcionalmente en las dos primeras piezas, en la última pieza, sin embargo, repetidas veces, y alcanza con ello un aumento paulatino de dificultad para los alumnos.

El himno siguiente Ave maris stella $\mathrm{V}$ de Hernando adopta el motivo citado y, resumiblemente, por esa razón, aparece en este lugar de la colección. 


\section{Himno VIII Pange lingua I}

\section{Ejemplo 4.}

Himno VIII

Pange lingua I
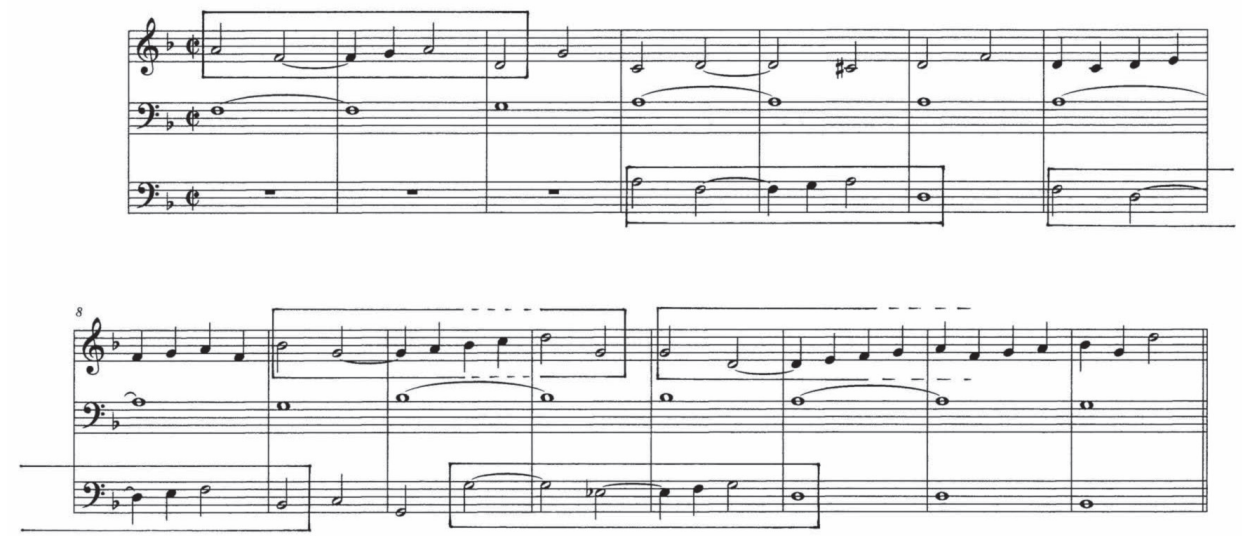

Se puede definir el himno VIII sobre el Pange lingua I, con razón, como monotemático. El motivo inicial, que anticipa las primeras notas del canto llano, es casi omnipresente, y aparece encadenado sólo en forma ligeramente transformada.

\section{Ejemplo 5.}

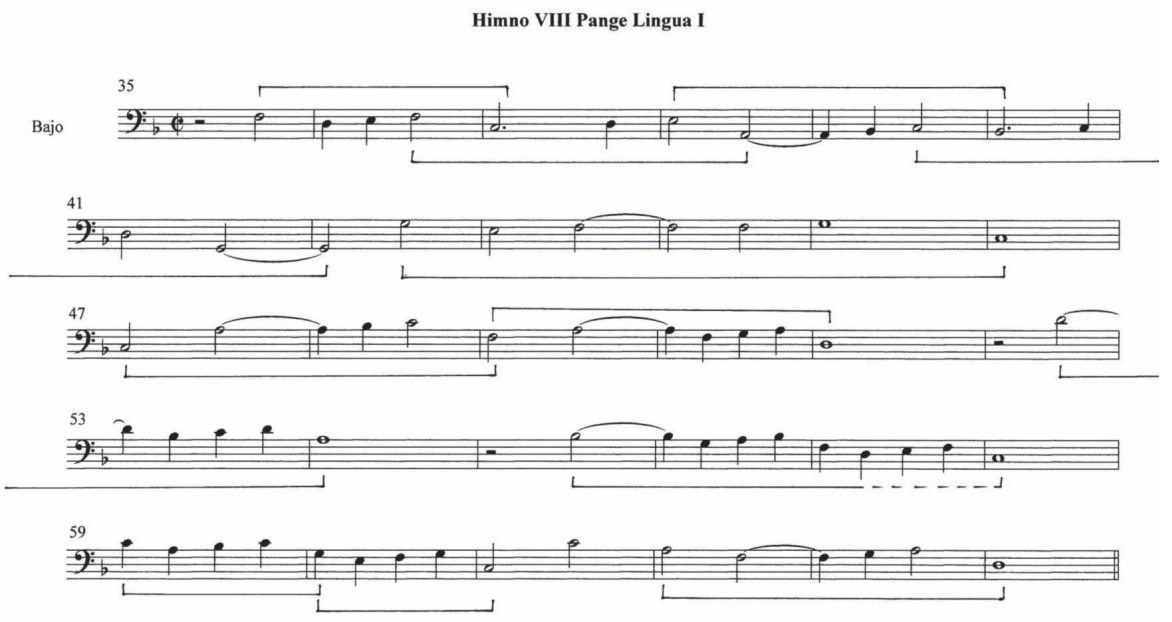


En esto, se aprende lo flexiblemente que se pueden tratar los motivos, tal vez mucho más, si son cortos y concisos. Cabezón consigue una enorme multiformidad en los detalles, sin que la composición se vuelva muy exigente en la técnica de ejecución. Sólo esporádicamente aparecen en las dos voces, que se imitan, movimientos simultáneos de negras.

\section{Las composiciones a cuatro voces. (La salmodia para principiantes) Versos del primer tono (1-4)}

En los versos a cuatro voces, se encuentran las composiciones más cortas, que Cabezón ha legado a la posteridad. Varios de estos versos minimalistas constan solamente de 13 compases y, con esto, son mucho más cortos que las composiciones del principio de la colección (Dúo I: 82, Dúo II: 43, Dúo III: 32 compases) y así son sumamente adecuados para hacer comprensible a los principiantes la estructura compositiva y, a la vez, dirigirlos hacia la ejecución de música imitativa a cuatro voces. Todos los versos están rigurosamente divididos en dos partes, como declara la nota del comienzo: „Estos versos son para los que comiençan, y de cada uno pueden hacer dos, quando quisieren acortar [...]“ El canto llano ${ }^{14}$, que recorre todas las cuatro voces en los versos del mismo tono, se presenta muy alargado, sobre todo, en la primera mitad, y aproximadamente hacia la mitad de los versos, la primera serie de sujetos, en las voces restantes, termina con una clausula.

\section{Ejemplo 6}

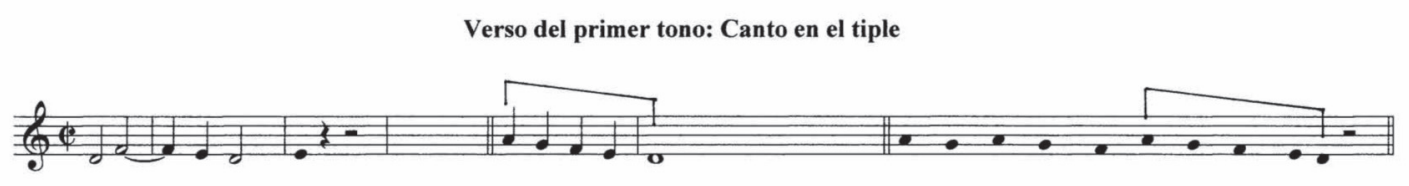

Verso del primer tono: Canto en el contralto

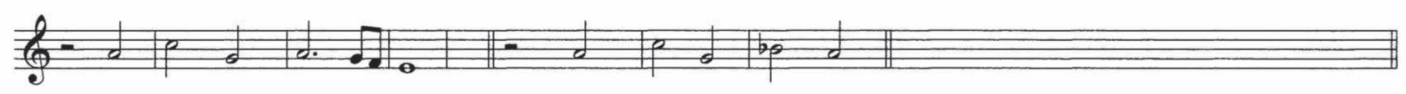

Verso del segundo tono: Canto en el contralto

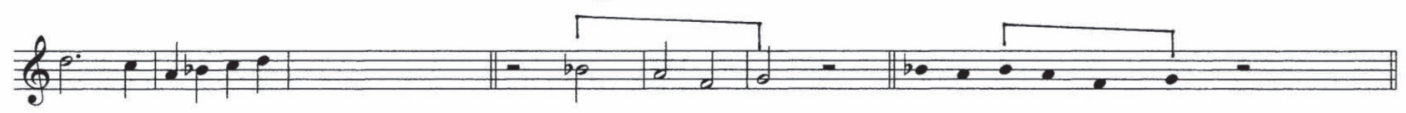

14 Esta misma estructura se encuentra en la serie de elaboraciones del Kyrie Rex virginum (vol. 2, p. 40 ss.), quedando aquí intacto el canto llano en cada segundo Kyrie y no incluído en la imitación. 
Ya que los sujetos están formulados cortos y sencillos, las semejanzas entre ellos o con el canto llano son también, estadísticamente, más probables, que en las melodías más extensas. Incluso, cuando dentro de los versos no se percibe ninguna sistemática, la coincidencia del segundo sujeto del ejemplo inicial con la segunda mitad del canto llano (fragmentos de escalas descendentes) es, probablemente, algo más que casual. En el segundo verso (canto llano en el contralto), el segundo sujeto se deriva claramente del primero, como sucede a menudo en Cabezón. Igualmente los sujetos del verso del segundo tono (canto llano en el contralto) son afines, pero adoptan adicionalmente un marcado movimiento del canto llano: el característico floreo final de tono salmódico.

Tales relaciones entre ambos sujetos, entre sujeto y canto llano, pero también entre sujetos de distintos versos (por ejemplo: segundo sujeto de xv, 3 y ambos sujetos de XVI, 2) se encuentran a menudo, al menos con mayor frecuencia, que permite la casualidad. Tales relaciones muestran la cercanía entre composición e improvisación, ya que, en el segundo caso, también la selección y agrupación de modelos preexistentes, pero mucho más plásticos, dentro de una totalidad lógica, sigue siendo el método elegido. Aprender a improvisar consiste, por un lado, en apropiarse de un gran depósito de modelos y tenerlo a disposición. Por otro, hay que ejercitar la habilidad de colocar los modelos en un plano integral más o menos premeditado, es decir, colocarlo de un modo más o menos espontáneo, para que resulte un satisfactorio efecto total. Las 32 piezas de la salmodia, en cualquier caso, son joyas compositivas e integran un microcosmos de un cuerpo de composiciones, diáfanamente estructuradas como el cristal, ricas en variantes y cíclicas, no sólo a causa de las fórmulas salmódicas, en las que el alumno puede aprender ingeniosamente y el experto complacerse.

Las dos composiciones del comienzo del Libro de cifra nueva de Venegas, que se atribuyen, debido a la indicación "Antonio" a Antonio de Cabezón, resultan claramente inferiores a aquellas. La composición inicial a dos voces sobre el Pange lingua no ofrece en absoluto trabajo motívico, aunque Cabezón lo ha realizado en otras composiciones a dos voces sobre un canto llano. En los himnos a dos voces sobre el Ave maris stella (vol. 1, 4-7) por ejemplo, imita dentro de la voz figurada añadida al canto llano, dando por resultado una textura oculta a tres voces, en la cual quasi se añaden dos voces complementarias. En el himno sobre Te lucis ante terminum (vol. 1, 8) empieza incluso imitativamente. Nada de eso puede hallarse en el citado Pange lingua.

Kastner excusa la calidad inferior de las composiciones del Libro de cifra nueva, que se atribuyen a Cabezón, por su referencia al grupo de destino 'principiantes' y a irreales prácticas comerciales de Venegas. Esto no me parece muy probable ${ }^{15}$. Como se ha demostrado en otras circumstancias, Cabezón siempre trabaja con suma precisión y alta calidad, nunca con negligencia. La declaración de Kastner manifiesta también un menosprecio al trabajo pedagógico-musical, que sería para Cabezón muy atípico, también considerando lo que nos ha legado su hijo Hernando. La quinta composición de la colección de

15 FROTSCHER, Gotthold: Geschichte des Orgelspiels und der Orgelkomposition, Berlin 1935. p. 259 tampoco considera a Cabezón como el autor. Por el contrario véase: Anglès, Higinio: La música en la Corte de Carlos V. Con la transcripcion del „Libro de cifra nueva para tecla, harpa y vihuela“ (Alcala de Henares, 1557). [compilado por] Luys Venegas de Henestrosa. Barcelona, CSIC, 1965. vol. II Música, pp. 169. 
Venegas "Sobre el canto llano de la alta“ podría proceder más bien de Cabezón. Pero también estamos aquí en un punto muerto (c. 45ss.), en efecto, contiene también pasajes muy densos (estrechos c. 37ss. y $85 s s$. , secuencia c. 112).

\section{EL PROBLEMA DE LA VALORACIÓN DEL NIVEL ARTÍSTICO}

No deben buscarse en textos científicos declaraciones sobre la calidad de las composiciones, porque, como juicios valorativos, tienen que referirse a sistemas individuales de valor, ofrecen apuestas y, por eso no son objectivables. Intentaré consecuentemente solucionar el problema por la vía de indicios, al menos parcialmente. Las pruebas autoritarias son obsoletas, opiniones de científicos, que, no obstante, se han ocupado detenidamente con la materia, son dignas de tenerse en consideración, y si señalan una dirección, muestran al menos una referencia.

Apel ${ }^{16}$ escribe sobre las composiciones "para principiantes": "With their brevity and proportionate simplicity in mind, and considering also such designations as Salmodia para principiantes ("Psalter for Beginners"), musical scholars like Ritter (in his Geschichte des Orgelspiels) and Seiffert (in his Geschichte der Klaviermusik) have designated these compositions as instruction and exercise material. But such a characterization, even if historically correct, has disadvantages, since it can all to easily give rise to the mistaken notion that we are here dealing with music of little artistic value, music just good enough for pedagogical purposes. In actual fact, these little compositions [...] are masterpieces of fully mature art-creations that contain within a few measures a combination of such fullness and force of compression as only a Bach knew how to unfold." En "Geschichte der Orgel- und Klaviermusik bis 1700” [Historia de la música para tecla hasta 1700] cita el himno a dos voces Te lucis (I/8) y escribe también con referencia a esta pieza lo siguiente: "A las melodías canto llano, rigurosamente formuladas, se unen voces contrarias contrapuntísticas, cuya vivacidad interior y expresividad permiten reconocer en cada momento la mano de un gran maestro. Así aparecen composiciones, que figuran entre los testimonios más impresionantes de la vigencia artística del estilo del cantus planus... Es digna de admiración la vida rica, que se desarrolla dentro de un marco tan sencillo..." Apel ${ }^{17}$

Sobre los versos, comenta Apel ${ }^{18}$ : "Con toda su brevedad y a pesar de ser escritos "para principiantes' (lo que podría proceder del editor) los versos de la salmodia pertenecen a las creaciones más importantes del maestro español de órgano... La concisión y expresividad de los sujetos es igual de admirable, que su elaboración en un marco tan reducido. »

En contraposición a las obras de Cabezón, fluctúa la apreciación de los ejemplos musicales de los otros dos tratados españoles contemporáneos. Häfner ${ }^{19}$ confirma la distinción de Bermudo, entre ejemplos de con-

\footnotetext{
16 APEL, Willi: "Early Spanish Musik for Lute and Keyboard Instruments", en: MQ 3/1934, p. 296.

17 Apel, Willi: Geschichte der Orgel- und Klaviermusik bis 1700, Kassel 1967. p. 124 y ss.

18 Ibidem, p. 129

19 HÄFNER, Roland: Die Entwicklung... op. cit.,p. 24.
} 
cierto y ejemplos didácticos: "Es verdad que los ejemplos añadidos al final del $4^{\circ}$. volumen están destinados expresamente a tocar, pero no deben ser considerados como ejercicios.“ Artigas Pina expone en el proemio a la Declaración de Bermudo ${ }^{20}$ : „Hay otras obras musicales para teclado insertas en la Declaración, pero escritas no para ser tañidas, sino desde un punto de vista absolutamente didáctico." Otras manifestaciones a la Declaración de Bermudo realzan el carácter didáctico, implican, sin embargo, la ausencia de calidad artística Kinkeldey ${ }^{21}$, En cuanto al Arte de tañer Fantasia de Tomás de Sancta María, Apel ${ }^{22}$ observa „Las numerosas piezas que se ofrecen para aclarar las diversas explicaciones, son ejemplos clásicos correctamente escritos, pero sin notable originalidad." Frotscher ${ }^{23}$ tampoco les reconoce calidad artística alguna: "Los muchos ejemplos que pone San[c]ta María, son ejemplos didácticos de estilo muy simple.” Por el contrario, Kinkeldey ${ }^{24}$ : „Y a pesar de la sencillez de las piezas, casi siempre son cantables e interesantes en todas las voces.“

\section{CONCLUSIONES}

Las composiciones ,para principiantes“ son muy variadas, es decir, presentan diversas posibilidades de tratar el material compositivo, y todas ellas muestran su individualidad. Por tanto, no se cumple mecánicamente el principio "de lo sencillo a lo dificil", como conocemos por muchos métodos, sobre todo del siglo XIX, sino que Cabezón, en primer plano, concibe las posibilidades, esboza los espacios de juego y las libertades, en vez de traspasar demasiado sistemáticamente un espacio limitado por reglas. Este segundo principio, muy didáctico y actual, de posibilitar el aprendizaje, en vez de guiar hacia una meta predefinida, distingue el método de Cabezón del de sus contemporáneos y de muchos métodos de tiempos posteriores. También es el requisito indispensable, para que nazca arte dentro un margen didáctico.

Todos los ejemplos de la parte didáctica de las «Obras...» están elaborados ingeniosamente, en algunos casos, debido a la brevedad, incluso son sumamente densos y homogéneos, es decir con un mínimo de material motívico y un máximo de variada formulación. Con las Obras de música para tecla, arpa y vihuela de Antonio y Hernando de Cabezón tenemos en manos uno de los ejemplos más antiguos de método instrumental, que reúne la lógica didáctica con el alto nivel artístico. La distancia de los métodos contemporáneos para tecla, respecto a la calidad, justifica, sin embargo, la valoración, que con este método se nos ha puesto en las manos, el primer ejemplo de música didáctica para tecla con valor artístico - 150 años antes de las correspondientes obras de Johann Sebastian Bach y 250 años antes del Album para la juventud de Robert Schumann.

Recibido: 12/08/2010

Aceptado: 03/06/2011

20 Artigas Pina, Javier: Estudio y edición de Fray Juan Bermudo: Obras para teclado (Cuadernos de Daroca II), Zaragoza 2005. p. 27

21 KinKeldey, Otto: Orgel und Klavier in der Musik des 16. Jahrhunderts, Leipzig 1910. pp. 10 y 136

22 APEL, Willi: Geschichte der Orgel- und Klaviermusik bis 1700, Kassel 1967. p. 201

23 Frotscher, Gotthold: Geschichte des Orgelspiels... op. cit. p. 251

24 KinKeldeY, Otto: Orgel und Klavier... op. cit., p. 136 


\section{BIBLIOGRAFÍA}

Anglés, Higinio “Antonio de Cabezón: su vida y su obra”, en: AnM 21, 1966, p. 3-16.

Apel, Willi "Early Spanish Musik for Lute and Keyboard Instruments", en: MQ3/1934, pp. 289-301. Apel, WiLli Geschichte der Orgel- und Klaviermusik bis 1700, Kassel 1967.

Apel, Willi Art. "Orgelmusik“, Sección III 16. Jahrhundert, en: $M G G$ nueva ed., vol. 7, columnas 1065-1069.

Artigas Pina, Javier Estudio y edición de Fray Juan Bermudo: Obras para teclado (Cuadernos de Daroca II), Zaragoza 2005.

Cabezón, Antonio de Obras de música para tecla, arpa y vihuela..., Madrid, 1578, ed. Felipe Pedrell, nueva edición Higinio Anglés, Barcelona 1966.

Ehrenforth, Karl HeInz Verstehen und Auslegen (Schriftenreihe zur Musikpädagogik), Frankfurt am Main, 1971.

Finscher, Ludwig / LeOpold, Silke „Volkssprachliche Gattungen und Instrumentalmusik“, en: Ludwig Finscher (ed.): Die Musik des 15. und 16. Jahrhunderts (Neues Handbuch der MuWi 3, 2), Laaber 1990, pp. 437-587.

Frotscher, GotTHOLd Geschichte des Orgelspiels und der Orgelkomposition, Berlin 1935.

HÄFner, Roland Die Entwicklung der Spieltechnik und der Schul- und Lehrwerke für Klavierinstrumente (Schriftenreihe des musikwissenschaftlichen Seminars der Universität München, 2), Munich 1937.

Howell, A. C. „CABezón: An Essay in Structural Analysis“, in: $M Q$ 50,1964, pp. 18-30.

Jambou, Louis Art. “Antonio de Cabezón”, en: The New Grove, vol. 4, 22001, p. 764.

Kastner, Macario Santiago Antonio und Hernando de Cabezón. Tutzing 1977.

Kastner, Macario Santiago Art. “Cabezón”, en: The New Grove Dictionary, vol. 3, Londres ${ }^{1} 1980$, p. 572.

Kastner, Macario Santiago "Sobre las diferencias de Antonio de Cabezón contenidas en las obras de 1578“, en: RdMc 4, 1981, p. 213-235.

KinKeldey, Otto Orgel und Klavier in der Musik des 16. Jahrhunderts, Leipzig 1910.

Kugler, Michael Die Musik für Tasteninstrumente im 15. und 16. Jahrhundert (Taschenbücher zur Musikwissenschaft, 41), Wilhelmshaven 1975.

MahleRt, Ulrich „Pädagogische Musik, en: Instrumental- und Vokalpädagogik“, ed. Chr. Richter (Handbuch der Musikpädagogik 2), Londres 1993, pp. 197-234.

Reimann, Margarete „Die Überlieferung von Antonio de Cabezóns Klavierwerken und ihre Spiegelung in seinen Diferencias“, en: AnM 21, 1966, pp. 27-38.

Roig-Francolí, Miguel A. "En torno a la figura y la obra de Tomás de Sancta María: Aclaraciones, evaluaciones y relación con la música de Cabezón“, en: RdMc 15, 1992, p. 55-85. 
SCHRADE, LEO Die handschriftliche Überlieferung der ältesten Instrumentalmusik, Lahr 1931.

Schwarting, JutTa und Heino Art. "Klavier", en: Instrumental- und Vokalpädagogik, ed. Christoph Richter (Handbuch der Musikpädagogik 3), Londres 1994, pp. 313-334.

Terni, Clemente "Ricordo de Antonio de Cabezón“, en: Chigiana 23, 1966, pp. 25-41.

Venegas de Henestrosa, Luys Libro de Cifra Nueva para tecla, harpa y vihuela, ed. Higinio Anglés (La música en la Corte de Carlos V), Barcelona 1965. 
